\title{
Anti-N-Methyl-D-Aspartate Receptor Encephalitis and Ovarian Teratoma: A Case Report
}

\author{
Sonoko Kubota ${ }^{\text {a }}$, Tatsuo Fuchigamia, e, Emiko Momoki ${ }^{\text {a }}$, Reina Hoshi ${ }^{\mathrm{b}}$, Takeshi Furuya ${ }^{\mathrm{b}}$, \\ Yoshiaki Kusumi $^{c}$, Wakako Ishii ${ }^{\text {a }}$, Yuki Kawamura ${ }^{a}$, Yukihiko Fujitad, \\ Shori Takahashi ${ }^{a}$
}

\begin{abstract}
Anti-N-methyl-D-aspartate receptor (anti-NMDAR) encephalitis is often associated with ovarian teratomas in young women. However, children are less likely to develop this condition. We herein report a very rare case of anti-NMDAR encephalitis and an ovarian teratoma in a young girl. A previously healthy 9 -year-old girl presented with convulsions and unconsciousness. Her cerebral spinal fluid was positive for anti-NMDAR antibody. She underwent immunotherapy, and although her clinical course improved, she did not fully recover. A right ovarian teratoma was then found and removed, and she became negative for anti-NMDAR antibody. She exhibited almost full recovery and no relapse. Tumor resection is recommended in the treatment of anti-NMDAR encephalitis. A tumor search should be actively carried out in patients aged $<12$ years.
\end{abstract}

Keywords: Anti-NMDAR encephalitis; Ovarian tumor; Teratoma; Young girl; Child

\section{Introduction}

Encephalitis associated with antibodies to the N-methyl-D-aspartate receptor (NMDAR) was first reported in 2007 by Dalmau et al [1]. Many retrospective and prospective studies have since been published, indicating the differences between children and adults with anti-NMDAR encephalitis [2-5]. Anti-NMDAR en-

Manuscript submitted November 5, 2016, accepted May 19, 2017

aDepartment of Pediatrics and Child Health, Nihon University School of Medicine, Tokyo, Japan

bDepartment of Pediatric Surgery, Nihon University School of Medicine, Tokyo, Japan

'Department of Pathology, Nihon University School of Medicine, Tokyo, Japan

${ }^{\mathrm{d}}$ Center for Institutional Research and Medical Education, Nihon University School of Medicine, Tokyo, Japan

${ }^{e}$ Corresponding Author: Tatsuo Fuchigami, Department of Pediatrics and Child Health, Nihon University School of Medicine, 30-1 Oyaguchi-Kamicho, Itabashi-ku, Tokyo 173-8610, Japan. Email: fuchigami.tatsuo@nihon-u.ac.jp

doi: https://doi.org/10.14740/ijcp260w cephalitis is often associated with tumors. One of the most important age-related differences is the frequency of the underlying neoplasm. The paraneoplastic rate is high in female patients aged $>12$ years, and younger patients are reportedly less likely to develop tumors; their clinical courses are rarely described [2-5].

We herein report a very rare case involving a young girl with anti-NMDAR encephalitis and an ovarian teratoma.

\section{Case Report}

A previously healthy 9-year-old girl was transferred to our care at the Department of Pediatrics, Nihon University School of Medicine, Itabashi Hospital, Tokyo. She presented with nausea and had been spitting saliva repeatedly for 7 days before admission to the prior hospital, where she was diagnosed with acetonemic vomiting. Her laboratory blood tests showed metabolic acidosis, ketosis, and low glycemia. Brain magnetic resonance imaging (MRI) showed no abnormalities. She underwent fluid infusion, and her laboratory data improved; however, her dysphagia persisted. She developed recurrent convulsions and unconsciousness on day 11 , and she was transferred to our hospital on day 13 . Her developmental history was normal, and she had no history of seizures. Her family history was unremarkable. There was no recent drug exposure, immunization, or infection. Menarche had not yet been observed.

On admission, she had an axillary body temperature of $37.5^{\circ} \mathrm{C}$, heart rate of 82 beats $/ \mathrm{min}$, blood pressure of $110 / 70$ $\mathrm{mm} \mathrm{Hg}$, and respiratory rate of 22 breaths $/ \mathrm{min}$. She was able to speak, but her speech was slower and more childish than before presentation. She had delirium with a Glasgow coma scale score of E4 V4 M6. A neurologic examination was normal except for a reduced level of consciousness. Her chest and abdomen showed no abnormal findings.

Laboratory blood test results were normal, including a full blood count, serum electrolytes, total protein, albumin, blood urea nitrogen, creatinine, aspartate aminotransferase, alanine aminotransferase, lactate dehydrogenase, alkaline phosphatase, creatine kinase, glucose, C-reactive protein, and anti-nuclear antibody. Her cerebrospinal fluid (CSF) showed lymphocytic pleocytosis of 16 nucleated cells $/ \mathrm{mm}^{3}$ with $100 \%$ lymphocytes and normal glucose and protein levels. Myelin basic protein and oligoclonal bands of immunoglobulin $\mathrm{G}$ in the CSF were negative. Herpes simplex virus DNA was not detected by real 

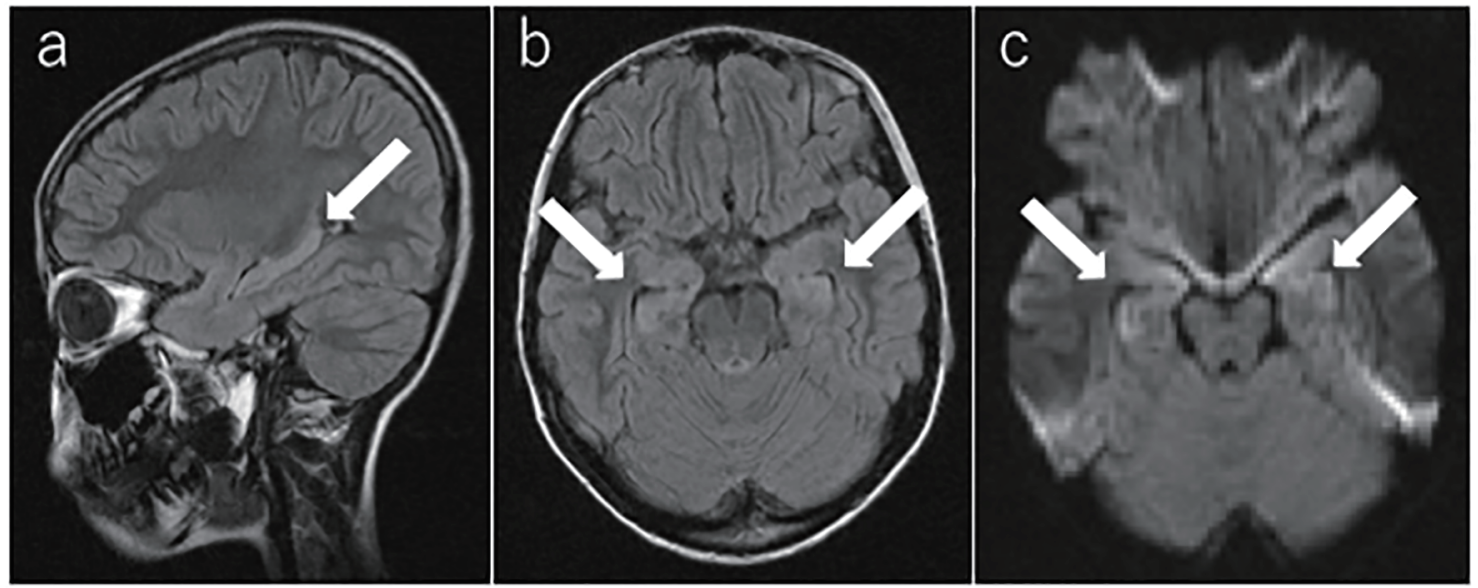

Figure 1. Brain magnetic resonance imaging. (a, b) T2-weighted fluid-attenuated inversion recovery image and (c) diffusionweighted image on the second day of admission (day 14). The arrow shows the high-intensity areas in the bilateral medial temporal lobes and hippocampus.

time PCR. Bacterial infection was not suspicious; therefore, cultivation of the CSF was not performed. Electroencephalography (EEG) showed generalized slow waves and no definitive epileptic discharges. Brain MRI was performed on the second day of admission (day 14). T2-weighted fluid-attenuated inversion recovery (FLAIR) and diffusion-weighted images showed hyperintense lesions in the bilateral medial temporal lobes and hippocampus (Fig. 1).

These findings strongly suggested acute limbic encephalitis. A 3-day treatment course of methylprednisolone pulse therapy $(30 \mathrm{mg} / \mathrm{kg} /$ day) and acyclovir $(60 \mathrm{mg} / \mathrm{kg} /$ day $)$ was promptly started. She showed agitation and confusion throughout the first night of admission to our hospital. She presented with her eyes wide open, spit saliva repeatedly, exhibited orofacial dyskinesia and stereotyped movement of the mouth, parroted others' words, and muttered meaningless words. She was given $400 \mathrm{mg} / \mathrm{kg} /$ day of intravenous immunoglobulin for 5 days starting on the second day after admission. She underwent three courses of methylprednisolone pulse therapy. Continuous intravenous midazolam was administered to calm her down. She required no respiratory support such as mechanical ventilation. Her symptoms improved gradually, and the continuous midazolam therapy was discontinued on day 27. Oral risperidone (1 mg/day) was started while the intravenous mi- dazolam was being decreased. However, her clinical features, including her abnormal behavior, psychomotor agitation, and memory deficit, had not yet fully recovered.

One month after admission, her CSF was found to be positive for antibodies to the NR1/NR2 heteromers of the NMDAR. Abdominal ultrasonography was performed to check for ovarian tumors and revealed a right ovarian tumor with no internal vascularity. On day 31, abdominal MRI also exhibited the right ovarian tumor (Fig. 2). On day 35, enhanced abdominal computed tomography showed calcific and fatty components within the tumor (Fig. 3). The patient was diagnosed with anti-NMDAR encephalitis with an ovarian tumor.

On day 49, laparoscopy-assisted resection of the right ovarian tumor was performed. Pathological examination revealed a $5.0 \times 3.5 \mathrm{~cm}$ mature ovarian cystic teratoma consisting of heterologous elements including central nervous system tissue, cerebellar tissue, choroid plexus, ganglion, skin, fat, sebaceous glands, hair follicles, cartilage, bone, and intestinal epithelia (Fig. 4). The fluid within the resected teratoma was negative for antibodies to the NR1/NR2 heteromers of the NMDAR. On day 52, her full-scale intelligence quotient using the Wechsler Intelligence Scale for Children-Fourth Edition was 83.

The pleocytosis in the CSF disappeared upon discharge, and the patient became negative for anti-NMDAR antibodies
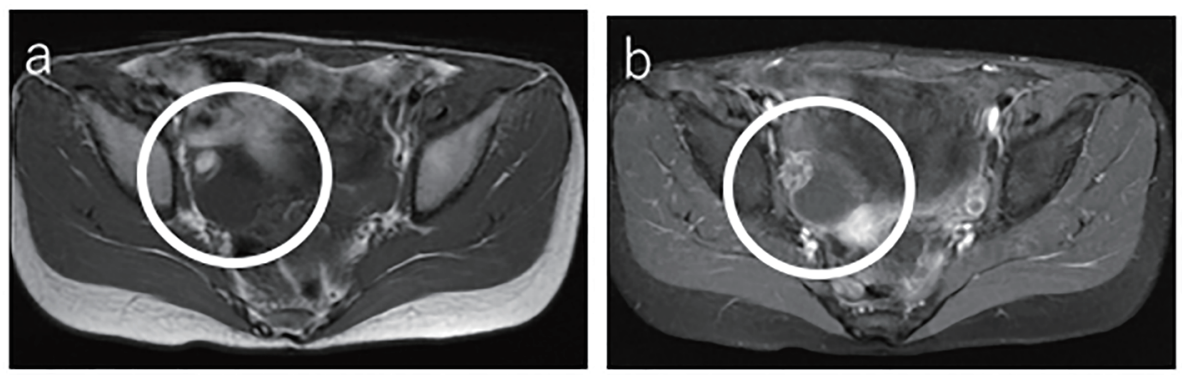

Figure 2. Abdominal magnetic resonance imaging. (a) T1-weighted fluid-attenuated inversion recovery image on day 31 showed the right ovarian tumor with a high-intensity cystic lesion. (b) Fat-saturated gadolinium-enhanced T1-weighted fluid-attenuated inversion recovery image showed a low-intensity lesion. 


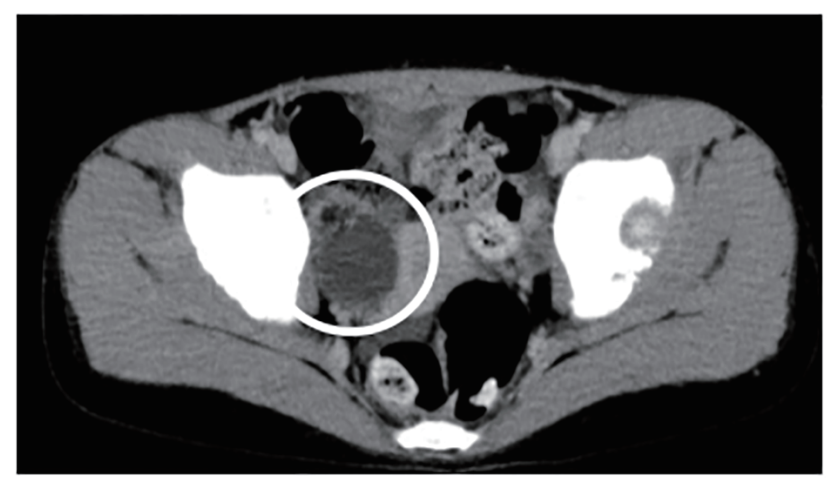

Figure 3. Enhanced abdominal computed tomography. On day 35, computed tomography showed the right ovarian tumor, including calcific and fatty components.

on day 71. EEG findings on day 76 showed that the slow waves disappeared and an $\alpha$ rhythm appeared. She was discharged from the hospital with slight neurological abnormalities (memory deficit and cognitive disturbance). Because of the physical restraint required during hospitalization, her muscle strength had weakened, but she gradually regained function through rehabilitation. Oral prednisolone therapy was continued after discharge and gradually tapered.

About 1 year after discharge, her intelligence quotient had improved. Her full-scale intelligence quotient, verbal comprehension index, perceptual reasoning index, working memory index, and processing speed index using the Wechsler Intelligence Scale for Children-Fourth Edition were 90, 90, 104, 82, and 91, respectively. She exhibited full recovery except for a reduced working memory index; overall, she had a good clinical outcome with no relapse.

\section{Discussion}

Anti-NMDAR encephalitis is often associated with ovarian teratomas in young women, but children are less likely to de- velop this condition, especially those aged $<12$ years [2-5]. Titulaer et al [2] reported that the median age at disease onset was 21 years, and $81 \%$ of 580 patients with anti-NMDAR encephalitis were female. The disease predominated in young female patients. The frequency of an underlying tumor was $38 \%$. A high proportion $(97 \%)$ of all patients with tumors were female, but a very low proportion $(6 \%)$ were aged $<12$ years. Most (94\%) of the tumors were ovarian teratomas [2]. The disease predominates in young female patients, but the difference in the presence of tumors between children and adults remains unclear. However, identification of which patients are likely to have tumors is important in the treatment of anti-NMDAR encephalitis [6]. Therefore, we have herein described the clinical course of a young girl ( 9 years of age) with anti-NMDAR encephalitis and an ovarian teratoma in this rare report.

With respect to the clinical features of anti-NMDAR encephalitis, about $70 \%$ of affected patients have prodromes consisting of non-specific symptoms such as headache, fever, nausea, vomiting, diarrhea, or upper respiratory tract symptoms. Within 2 weeks, patients develop psychiatric and/or neurologic symptoms [2]. Our patient developed nausea first, and her condition gradually worsened until she exhibited abnormal behavior and cognition, memory disturbance, childish speech, seizures, and loss of consciousness within 2 weeks.

Brain MRI findings are abnormal in $33-50 \%$ of affected patients $[1,2]$. On T2-weighted or FLAIR MRI, signal hyperintensity might be seen in the hippocampi, cerebellar or cerebral cortex, frontobasal and insular regions, basal ganglia, brain stem, and, infrequently, the spinal cord. These findings are usually mild or transient and can be accompanied by subtle contrast enhancement in the affected areas or meninges [7]. In the present case, T2-weighted FLAIR imaging and diffusionweighted imaging on the second day of admission (day 14) showed high-intensity areas in the bilateral medial temporal lobes and hippocampus.

EEG findings are abnormal in most patients (90\%), usually showing non-specific, slow, and disorganized activity sometimes with electrographic seizures [7]. Consistent with this, the EEG findings in our patient were characterized by non-specific

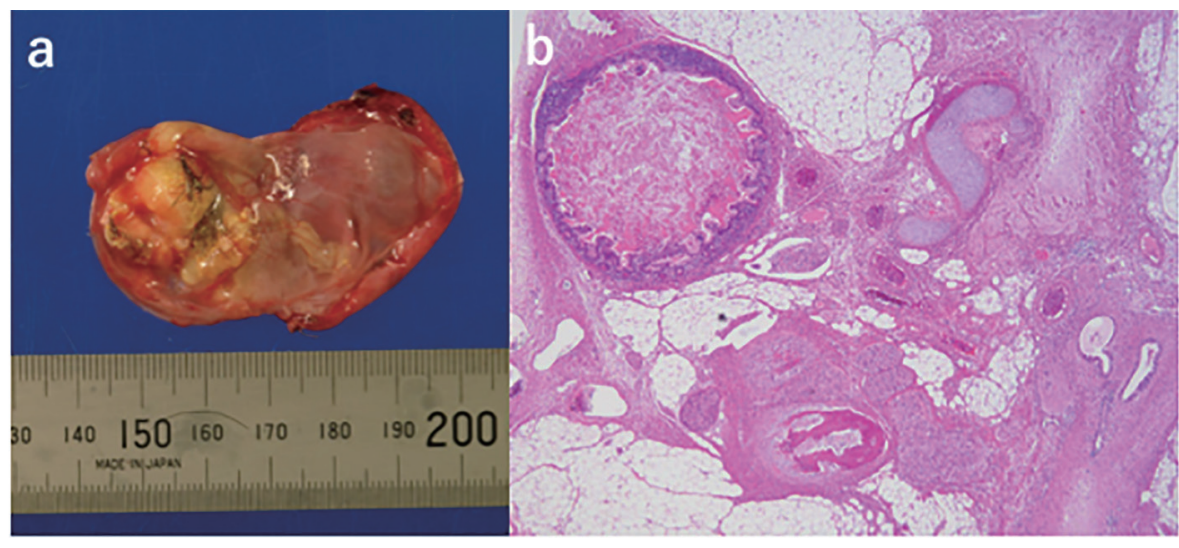

Figure 4. Pathological examination of the resected right ovarian tumor. (a) Macroscopic image of the right ovarian tumor after incision to reveal some hair (mature ovarian teratoma). A centimeter scale is shown under the specimen. (b) Histopathological image of the right ovarian tumor shows heterologous elements including central nervous system tissue, cerebellar tissue, choroid plexus, ganglion, skin, fat, sebaceous glands, hair follicles, cartilage, bone, and intestinal epithelia. 
slow and disorganized activity.

The CSF is initially abnormal in $80 \%$ of patients; it becomes abnormal later in the disease in most of the remaining patients [7]. Findings include moderate lymphocytic pleocytosis, a normal or mildly increased protein concentration, and, in $60 \%$ of patients, CSF-specific oligoclonal bands [6]. The CSF in our patient showed lymphocytic pleocytosis of 16 nucleated cells $/ \mathrm{mm}^{3}$ with $100 \%$ lymphocytes and normal glucose and protein levels. However, myelin basic protein and oligoclonal bands of immunoglobulin $\mathrm{G}$ were negative. The other clinical symptoms and laboratory findings in our patient were almost identical to those described in the literature.

Management of anti-NMDAR encephalitis should initially focus on immunotherapy and the detection and removal of any teratomas [6]. Identification and removal of all teratomas should be promptly considered after the diagnosis of anti-NMDAR encephalitis [8]. The prognosis changes if the neoplasm is removed at the onset of the disease. Thus, even if the patient is younger than 12 years, the presence of a tumor should be determined as soon as possible. Dalmau et al [1] reported that eight teratomas contained nervous tissue and that five were NMDAR-positive. These findings indicate that the pathogenesis of anti-NMDAR encephalitis in these patients with ovarian teratomas was related to antigenic stimulation from the NMDAR in the ectopic nervous tissue contained within the teratoma. The ovarian teratoma was histologically confirmed in our patient, and neural elements were also identified. These findings support those reported by Dalmau et al [1].

In a recent study by Titulaer et al [2], the frequency of an underlying teratoma was significantly greater in female patients aged $\geq 12$ years than in young children and male patients (52\% versus $6 \%$, respectively). Therefore, if a tumor is not found, we should carefully consider the patient's age and sex to guide decision-making regarding the extent and frequency of tumor screening. In female patients aged $\geq 12$ years, a screening approach similar to that used for paraneoplastic syndromes is appropriate (e.g., MRI of the abdomen and pelvis every 6 months for 4 years); in young children and male patients, however, the need for repeat screening is unclear [2].

Our patient exhibited a partial response to steroid pulse therapy and immunoglobulin therapy. Because she was a 9-year-old patient, we did not initially assume that an active tumor was present. However, additional examination finally revealed a tumor on day 31 . Further clinical improvement was achieved after tumor resection.

In conclusion, we have reported a case involving a 9-yearold girl with anti-NMDAR encephalitis and an ovarian teratoma. Anti-NMDAR encephalitis with a tumor is rare in patients aged $<12$ years. Tumor resection is recommended in the treatment algorithm of anti-NMDAR encephalitis. Even if the patient is aged $<12$ years, a tumor search should be actively carried out.

\section{Acknowledgments}

We thank Prof. Keiko Tanaka from the Department of Neu- rology, Kanazawa Medical University, Uchinada, Ishikawa for performing the anti-NMDAR antibody measurements.

\section{Author Contributions}

S. Kubota, E. Momoki, R. Hoshi, T. Furuya, W. Ishii, and Y. Kawamura provided medical care. S. Kubota drafted the manuscript. Y. Kusumi performed the pathological diagnosis of the ovarian tumor. T. Fuchigami designed the outline of the manuscript and provided conceptual advice. Y. Fujita and S. Takahashi supervised the whole study. All authors read and approved the final manuscript.

\section{Conflicts of Interest}

The authors declare no conflicts of interest.

\section{References}

1. Dalmau J, Tuzun E, Wu HY, Masjuan J, Rossi JE, Voloschin A, Baehring JM, et al. Paraneoplastic anti-Nmethyl-D-aspartate receptor encephalitis associated with ovarian teratoma. Ann Neurol. 2007;61(1):25-36.

2. Titulaer MJ, McCracken L, Gabilondo I, Armangue T, Glaser C, Iizuka T, Honig LS, et al. Treatment and prognostic factors for long-term outcome in patients with antiNMDA receptor encephalitis: an observational cohort study. Lancet Neurol. 2013;12(2):157-165.

3. Armangue T, Titulaer MJ, Malaga I, Bataller L, Gabilondo I, Graus F, Dalmau J. Pediatric anti-N-methyl-D-aspartate receptor encephalitis-clinical analysis and novel findings in a series of 20 patients. J Pediatr. 2013;162(4):850-856 e852.

4. Florance NR, Davis RL, Lam C, Szperka C, Zhou L, Ahmad S, Campen CJ, et al. Anti-N-methyl-D-aspartate receptor (NMDAR) encephalitis in children and adolescents. Ann Neurol. 2009;66(1):11-18.

5. Wright S, Hacohen Y, Jacobson L, Agrawal S, Gupta R, Philip S, Smith M, et al. N-methyl-D-aspartate receptor antibody-mediated neurological disease: results of a UK-based surveillance study in children. Arch Dis Child. 2015;100(6):521-526.

6. Dalmau J, Lancaster E, Martinez-Hernandez E, Rosenfeld MR, Balice-Gordon R. Clinical experience and laboratory investigations in patients with anti-NMDAR encephalitis. Lancet Neurol. 2011;10(1):63-74.

7. Dalmau J, Gleichman AJ, Hughes EG, Rossi JE, Peng X, Lai M, Dessain SK, et al. Anti-NMDA-receptor encephalitis: case series and analysis of the effects of antibodies. Lancet Neurol. 2008;7(12):1091-1098.

8. Seki M, Suzuki S, Iizuka T, Shimizu T, Nihei Y, Suzuki N, Dalmau J. Neurological response to early removal of ovarian teratoma in anti-NMDAR encephalitis. J Neurol Neurosurg Psychiatry. 2008;79(3):324-326. 